\title{
Axion clockworks from heterotic M-theory: the QCD-axion and its ultra-light companion
}

\author{
Sang Hui Im, ${ }^{a}$ Hans Peter Nilles ${ }^{b}$ and Marek Olechowski ${ }^{c}$ \\ ${ }^{a}$ Department of Physics, Pusan National University, \\ Busan 46241, Korea \\ ${ }^{b}$ Bethe Center for Theoretical Physics and Physikalisches Institut der Universität Bonn, \\ Nussallee 12, 53115 Bonn, Germany \\ c Institute of Theoretical Physics, Faculty of Physics, University of Warsaw, \\ ul. Pasteura 5, 02-093 Warsaw, Poland \\ E-mail: imsanghui@pusan.ac.kr, nilles@th.physik.uni-bonn.de, \\ Marek.Olechowski@fuw.edu.pl
}

ABSTRACT: A previously discussed clockwork mechanism within heterotic M-theory is applied to its axion landscape. We identify a unique candidate for a QCD-axion with a decay constant in the preferred "axion window" around $10^{11} \mathrm{GeV}$. It is accompanied by at least one ultra-light axion that couples predominantly to hidden sector gauge groups.

KEYwords: Beyond Standard Model, M-Theory, Superstrings and Heterotic Strings, Field Theories in Higher Dimensions

ArXiv EPrint: 1906.11851 


\section{Contents}

1 Introduction 1

2 Axion candidates 3

2.1 Weakly coupled heterotic string 3

2.2 Heterotic M-theory 6

3 Mixing $\quad 8$

3.1 Weakly coupled heterotic string 8

3.2 Heterotic M-theory 8

4 Decay constants and couplings $\quad 11$

$\begin{array}{ll}4.1 \text { Weakly coupled heterotic string } & 11\end{array}$

4.2 Heterotic M-theory 11

5 Towards a QCD axion $\quad 13$

6 Conclusions and outlook 16

$\begin{array}{ll}\text { A Axion couplings for } h_{(1,1)}=2 & 17\end{array}$

$\begin{array}{ll}\text { B Kaluza-Klein axions } & 19\end{array}$

\section{Introduction}

Axions have been considered in various areas of particle physics and cosmology, such as the solution to the strong CP-problem [1], the origin of inflation [2] and their contribution to dark matter and dark energy of the universe. These applications are typically connected to peculiar relations (hierarchies) of the mass scales under consideration. The QCD-axion as a solution of the strong CP-problem has to have a decay constant in the so-called "axion window" between $10^{9}$ and $10^{12} \mathrm{GeV}[3]$ much larger than the QCD scale; ${ }^{1}$ axionic inflation seems to require trans-Planckian excursions of the inflaton field [9, 10]. These scales could be chosen ad hoc, but in a consistent ultraviolet (UV) complete theory one would like to understand the origin of these scales and hierarchies as well as their relation to physical mass scales like the Planck mass, the weak (TeV) scale and the QCD scale.

A specific mechanism to create a hierarchy of axionic scales has been proposed in the framework of axionic (natural) inflation to create trans-Planckian axion decay constants via the alignment of (two) axions [11, 12], each with sub-Planckian decay constant. This alignment mechanism has been generalised to the multi-axion case [13] that allows the creation of an even larger hierarchy from moderate values of the fundamental parameters.

\footnotetext{
${ }^{1}$ Here we will assume the standard axion cosmology without consideration of late time entropy injection $[4,5]$ or stronger QCD phase in the early universe [6-8] which may relieve the upper bound $10^{12} \mathrm{GeV}$ on the QCD axion decay constant.
} 
Such a multi-axion approach has been used to create a large scale for the QCD axion (within the "axion window") starting from weak scale physics in the TeV-range [14].

A new challenge towards the creation of large hierarchies appears with the proposal of the so-called "relaxion mechanism" for a dynamical creation of the weak scale from the Planck scale [15]. Again, the mechanism of axion alignment was applied successfully [16]. It was subsequently pointed out that there is a similarity of the aligned axions and a "clockwork" mechanism [17]. As such, the multi-axion systems discussed here resemble approaches with accidental continuous symmetries (accions) through discrete symmetries [18] or the consideration of deconstructed extra dimensions [19, 20]. A next step considered the continuum limit $N \rightarrow \infty$ of aligned $N$-axion systems [21]. Discrete and continuous clockworks share some similarities but differ in the detailed interpretation as discussed in refs. [22-24]. While all these applications are very promising for the creation of hierarchies, there still remains the question of the embedding in a consistent ultraviolet completion. In previous work [25] we have considered the continuous clockwork mechanism in the framework of heterotic M-theory of Hořava and Witten [26]. ${ }^{2}$ For certain specific clockwork parameters we could accomodate a large hierarchy between the Planck scale and the weak $(\mathrm{TeV})$ scale.

In the present paper we investigate the options for the QCD-axion as a solution to the strong CP-problem within a heterotic M-theory clockwork scheme, i.e. we explore the possibilities to obtain an axion decay constant within the allowed "axion window", extending previous discussions of QCD-axions embedded in string theory [31]. Within the framework of the (weakly coupled) heterotic string it turns out to be very difficult to arrange for a large hierarchy between the Planck scale and the axion scale because these scales are strictly related through the value of the gauge coupling constants. This is different in heterotic M-theory (strongly coupled case) because of the appearance of warped extra dimensions. The gauge coupling constants on the visible and hidden brane are controlled by the volume of the compact six-dimensional space (Calabi-Yau (CY) manifold) on the respective brane. A hierarchy of scales could emerge in the situation of particular nonstandard embeddings where the volume of the hidden brane is larger than that of the visible brane. In our previous work [25] we have discussed this clockwork mechanism towards a solution of the hierarchy problem between the weak scale and the Planck scale. In the present paper we explore the mechanism for the explanation of the (more moderate) hierarchy between the Planck scale and the QCD-axion decay constant. We illustrate the mechanism in the simplest case of two axions known as the model-independent (MI) and the model-dependent (MD) axion [32]. This corresponds to the case of one Kähler modulus of the Calabi-Yau manifold $\left(h_{(1,1)}=1\right)$ and the clockwork parameter $\hat{c}^{2}=6[25]$. More general cases will be relegated to the appendix.

Our analysis clarifies that there is no possibility for an acceptable QCD-axion in weakly coupled heterotic string theory: the axion decay constants will always be of order of the

\footnotetext{
${ }^{2}$ As discussed in [24], the notion of continuous clockwork incorporates Randall-Sundrum (RS) model [27] and large extra dimension (LED) [28] as well as more general warped extra dimensional models, such as linear dilaton model [29]. The extra dimensions of heterotic M-theory [30] can be described in terms of continuous clockwork as well [25].
} 
string scale and thus far above the "axion window". The case of heterotic M-theory, however, looks more promising. The continuous clockwork mechanism discussed in ref. [25] allows a lowering of the relevant axion decay constant. The results of the present paper can be summarised as follows:

- There are various possibilities to lower axion decay constants to match the "axion window".

- Among them there is, however, only a unique candidate for a QCD axion where $M_{11}$ (the fundamental mass scale of M-theory) is lowered to a value not too far from that axion window. In fact, the axion decay constant $f_{a}$ of the QCD axion will be an order of magnitude smaller than $M_{11}$.

- This QCD axion is accompanied by at least one (hidden) ultra-light axion (ULA). Its decay constant is smaller than that of the QCD axion and it is essentially decoupled from the observable sector. Properties of such ULA(s) should be investigated thoroughly.

The paper is structured as follows. In section 2 we shall present the axion candidates, separately for the weakly coupled heterotic string and heterotic M-theory. Section 3 discusses the mixing between the various axion candidates and points out specific differences between the cases of string and M-theory. In section 4 we shall analyse the axion decay constants for the two separate cases. Section 5 is devoted to the analysis of candidates for a QCD axion. There we shall present the unique axion solution to the strong CP-problem. Section 6 will be devoted to conclusions and outlook. Some technical details and discussion of Kaluza-Klein (KK) axions that originate from the clockwork mechanism will be given in the appendices.

\section{Axion candidates}

In this section, we will review the axion candidates under consideration. We shall identify and compare the candidates in the standard 10-dimensional heterotic string theory (weakly coupled case) with those in 11-dimensional heterotic M-theory (strongly coupled case).

\subsection{Weakly coupled heterotic string}

The part of the low energy action of the heterotic string theory relevant for our discussion can be written $\mathrm{as}^{3}$

$$
\mathcal{S}_{10}=\frac{1}{2 \kappa_{10}^{2}} \int_{\mathcal{M}^{10}}\left(\star \mathcal{R}-\frac{\ell_{s}^{4}}{2} H \wedge \star H-\frac{\alpha^{\prime}}{4} \operatorname{tr} F \wedge \star F\right)-\int_{\mathcal{M}^{10}} B \wedge X_{8}+\ldots
$$

where $\kappa_{10}^{2}=e^{2 \phi}\left(\alpha^{\prime}\right)^{4} / 8$, the string tension $\alpha^{\prime}$ and length $\ell_{s}$ are related by $\alpha^{\prime}=\left(\ell_{s} / 2 \pi\right)^{2}$, $H$ is the field strength of the 2 -form field $B, F$ is the $E_{8} \times E_{8}$ gauge field strength. The first term is understood as the classical action of heterotic string theory, while the

\footnotetext{
${ }^{3}$ We adopt the conventions used in [31].
} 
second integral in the r.h.s. of the above action is necessary for the Green-Schwarz (GS) anomaly cancellation mechanism [33] with the 8-form constructed out of $F$ and $\mathcal{R} 2$-forms: $X_{8}=c_{1} \operatorname{tr} F^{4}+c_{2}\left(\operatorname{tr} F^{2}\right)^{2}+c_{3} \operatorname{tr} F^{2} \operatorname{tr} \mathcal{R}^{2}+\cdots$. It could be interpreted as a 1-loop quantum correction to the classical theory.

The field strength $H$ has to satisfy the modified Bianchi identity

$$
d H=-\frac{1}{16 \pi^{2}}(\operatorname{tr} F \wedge F-\operatorname{tr} \mathcal{R} \wedge \mathcal{R})
$$

for the theory to be supersymmetric and anomaly free. This leads to the following expression for $H$

$$
H=d B+\omega_{3},
$$

where

$$
\omega_{3} \equiv-\frac{1}{16 \pi^{2}}\left(\omega_{Y M}-\omega_{L}\right)
$$

with

$$
\omega_{Y M} \equiv \operatorname{tr}\left(A F-\frac{2}{3} A^{3}\right), \quad \omega_{L} \equiv \operatorname{tr}\left(\omega \mathcal{R}-\frac{2}{3} \omega^{3}\right)
$$

which are the Yang-Mills and Lorentz Chern-Simons 3-forms, respectively.

We are interested mainly in the axion fields in $\mathcal{N}=1$ SUSY theory in $4 \mathrm{D}$, so in order to get their definitions and interactions we compactify the 10D theory described by the action (2.1) on a compact 6D CY manifold $\mathcal{X}^{6}$. Thus, we assume $\mathcal{M}^{10}=\mathcal{M}^{4} \times \mathcal{X}^{6}$ with $\mathcal{M}^{4}$ being the $4 \mathrm{D}$ Minkowski space-time. For the reduction procedure, we expand $B$ and $\omega_{3}$ forms in harmonic $(1,1)$-forms $\omega_{i}^{(1,1)} \in H^{(1,1)}(X)\left(i=1, \ldots, h_{(1,1)}\right)$ on $\mathcal{X}^{6}$ :

$$
\begin{aligned}
B & =\frac{1}{2 \pi} \omega_{i}^{(1,1)} b^{i}(x)+\cdots, \\
\omega_{3} & =\frac{1}{2 \pi} \omega_{i}^{(1,1)} \wedge q^{i} A(x)+\cdots,
\end{aligned}
$$

where $b^{i}(x)$ and $A(x)$ are $4 \mathrm{D}$ fields and factors of $1 / 2 \pi$ are introduced to obtain $2 \pi$ periodicity of the resulting axions and $q^{i}$ are dimensionless numbers depending on the gauge bundles over $\mathcal{X}^{6}$. The important part of the 8 -form $X_{8}$ from the Green-Schwarz term is given by

$$
X_{8}=\omega^{(3,3)} \wedge q F(x)+\frac{1}{8 \pi}\left(\operatorname{tr}_{1} F \wedge F-\frac{1}{2} \operatorname{tr} \mathcal{R} \wedge \mathcal{R}\right) \wedge\left(\operatorname{tr}_{1} F \wedge F-\operatorname{tr}_{2} F \wedge F\right)+\cdots,
$$

where $\omega^{(3,3)}$ is the harmonic $(3,3)$ form on the CY space $\mathcal{X}^{6}, q$ is another dimensionless number determined from the gauge bundles over $\mathcal{X}^{6}$, and $\operatorname{tr}_{j}$ is trace in the $j$-th $E_{8}$ gauge group.

Using eqs. (2.6), (2.7) and (2.8) in the action (2.1), the relevant part of the resultant $4 \mathrm{D}$ action may be written in the form

$$
\mathcal{S}_{4, \text { axion }}=\mathcal{S}_{4, \mathrm{MI}}+\mathcal{S}_{4, \mathrm{MD}}
$$

with the first term in the r.h.s. given by

$$
\mathcal{S}_{4, \mathrm{MI}}=-\frac{\ell_{s}^{4} V_{X}}{2 \kappa_{10}^{2}} \int_{\mathcal{M}^{4}} \frac{1}{2} H \wedge \star H-\int_{\mathcal{M}^{4}} q B \wedge F .
$$


This part of the action describes dynamics of the 3 -form $H$ which in $4 \mathrm{D}$ may be dualized to a scalar. A way to dualize $H_{\mu \nu \rho},{ }^{4}$ is to enforce the Bianchi identity (2.2) by adding to $(2.10)$ a term with a 1 -form Lagrange multiplier. Then $H$ may be eliminated using its equation of motion first and action (2.10) may be rewritten in the form

$$
\mathcal{S}_{4, \mathrm{MI}}=-\frac{2 \kappa_{10}^{2}}{V_{X} \ell_{s}^{4}} \int_{\mathcal{M}^{4}} \frac{1}{2}(d a+q A) \wedge \star(d a+q A)+\frac{1}{16 \pi^{2}} \int_{\mathcal{M}^{4}} a\left(\operatorname{tr}_{1} F \wedge F+\operatorname{tr}_{2} F \wedge F\right),
$$

where $a$ is a scalar field to be identified as an axion. It couples the same way to both visible and hidden $E_{8}$ sectors and does not depend on the details of the compactification from $10 \mathrm{D}$ to $4 \mathrm{D}$. Thus, it is called the model independent (MI) axion. Loosely speaking, it corresponds to the pseudoscalar $B_{\mu \nu}$ and couples directly to the instantons in the classical action.

The second term in the r.h.s. of (2.9) reads

$\mathcal{S}_{4, \mathrm{MD}}=-\frac{\ell_{s}^{4}}{16 \pi^{2} \kappa_{10}^{2}} G_{i j} \int_{\mathcal{M}^{4}}\left(d b^{i}+q^{i} A\right) \wedge \star\left(d b^{j}+q^{j} A\right)+\frac{n_{i}}{16 \pi^{2}} \int_{\mathcal{M}^{4}} b^{i}\left(\operatorname{tr}_{1} F \wedge F-\operatorname{tr}_{2} F \wedge F\right)$,

where

$$
\begin{aligned}
G_{i j} & =\int_{\mathcal{X}^{6}} \omega_{i}^{(1,1)} \wedge \star \omega_{j}^{(1,1)}, \\
n_{i} & =-\frac{1}{16 \pi^{2}} \int_{\mathcal{X}^{6}} \omega_{i}^{(1,1)} \wedge\left(\operatorname{tr}_{1} F \wedge F-\frac{1}{2} \operatorname{tr} \mathcal{R} \wedge \mathcal{R}\right) .
\end{aligned}
$$

Action (2.12) describes $h_{(1,1)}$ axions $b^{i}$. They are called model dependent (MD) axions because, contrary to the MI axion $a$, their interactions depend on the details of the compactification via quantities $G_{i j}$ and $n_{i}$. Loosely speaking they can be identified with the pseudoscalars $B_{m n}$, with indices $m, n$ in the compact space. They have opposite sign couplings to visible and hidden $E_{8}$ gauge groups via the Green-Schwarz polynomial. Note that fields $a$ and $b^{i}$ are normalized to be $2 \pi$-periodic. In the 4D supergravity language axions are pseudoscalar components of chiral superfields. The MI axion belongs to the dilaton field $\mathcal{S}$ while the MD axions are parts of the moduli $\mathcal{T}^{i}$ (the overall modulus $\mathcal{T}$ in the case of $h_{(1,1)}=1$ ). At the classical level, these axions do not mix. Quantum corrections lead to a mixing of the axions as will be discussed in section 3 .

In the above discussions, we have ignored $q A$ and $q^{i} A$ for a while. In fact, some of the charges $q$ and $q^{i}$ do not vanish if there exist anomalous $\mathrm{U}(1)$ gauge symmetries in $E_{8}^{(1)} \times E_{8}^{(2)}$ under which the corresponding axions transform non-linearly. Such axions may be gauged away becoming longitudinal components of the gauge fields. Yet one can have remnant global U(1) $)_{\mathrm{PQ}}$ symmetries in matter sectors to deal with the strong CP problem $[31,32,34]$. In the next section, we will see that a similar structure appears for axions from the strongly coupled heterotic string, but the associated U(1) gauge symmetries are not directly related

\footnotetext{
${ }^{4}$ When referring to components we use the following conventions: indices from the middle of the Greek alphabet are tangent to Minkowski space $\mathcal{M}^{4}, \mu, \nu, \ldots=0,1,2,3$; indices from the middle of the Latin alphabet are tangent to $\mathrm{CY}$ space $\mathcal{X}^{6}, m, n, \ldots=5,6,7,8,9,10$; indices from the beginning of the Greek alphabet are tangent to $\mathcal{M}^{4} \times S^{1}, \alpha, \beta, \ldots=0,1,2,3,11$.
} 
to $E_{8}^{(1)} \times E_{8}^{(2)}$. They instead come from the bulk gauge invariance of the 3 -form $C$ of the 11D supergravity. This will give a clockwork structure for the axions in the strongly coupled theory.

\subsection{Heterotic M-theory}

The strongly coupled $E_{8} \times E_{8}$ heterotic string theory (heterotic M-theory) can be described in terms of $11 \mathrm{D}$ supergravity with the bosonic part of the action given by

$$
\begin{aligned}
\mathcal{S}_{11}= & \frac{1}{2 \kappa_{11}^{2}} \int_{\mathcal{M}^{11}}\left(\star \mathcal{R}-\frac{\ell_{11}^{6}}{2} G \wedge \star G-\frac{\ell_{11}^{9}}{6} C \wedge G \wedge G\right) \\
& -\frac{1}{8 \pi \kappa_{11}^{2}}\left(\frac{\kappa_{11}}{4 \pi}\right)^{2 / 3} \sum_{j=1}^{2} \int_{\mathcal{M}_{(j)}^{10}} \operatorname{tr} F_{(j)} \wedge \star F_{(j)},
\end{aligned}
$$

where $\kappa_{11}^{2}$ is related to fundamental 11D mass and length scales as follows: $\kappa_{11}^{2}=M_{11}^{-9}$ and $\kappa_{11}^{2}=\ell_{11}^{9} / 4 \pi$. $G$ is the field strengths of the 3 -form field $C,{ }^{5} F_{(j)}$ are the gauge field strengths of two $E_{8}^{(j)}(j=1,2)$ gauge groups living at the two $10 \mathrm{D}$ orbifold fixed hyperplanes $\mathcal{M}_{(j)}^{10}$.

In analogy to the weakly coupled heterotic string, the field strength $G$ has to satisfy a modified Bianchi identity which in this case takes the form

$$
\mathrm{d} G=J^{(1)} \wedge \delta\left(x^{11}\right) \mathrm{d} x^{11}+J^{(2)} \wedge \delta\left(x^{11}-\pi r_{11}\right) \mathrm{d} x^{11}
$$

with

$$
J^{(j)} \equiv-\frac{1}{8 \pi^{2}}\left(\operatorname{tr} F_{(j)} \wedge F_{(j)}-\frac{1}{2} \operatorname{tr} \mathcal{R} \wedge \mathcal{R}\right)=\mathrm{d} \omega_{3}^{(j)}
$$

where

$$
\omega_{3}^{(j)} \equiv-\frac{1}{8 \pi^{2}}\left(\omega_{Y M}^{(j)}-\frac{1}{2} \omega_{L}\right)
$$

while $\omega_{Y M}^{(j)}$ and $\omega_{L}$ are the Yang-Mills and Lorentz Chern-Simons 3-forms as in (2.5). The above Bianchi identity is solved when $G$ is defined as [26, 35]

$$
G=\mathrm{d} C+\omega_{4}
$$

where

$$
\omega_{4}=\omega_{3}^{(1)} \wedge \delta\left(x^{11}\right) \mathrm{d} x^{11}+\omega_{3}^{(2)} \wedge \delta\left(x^{11}-\pi r_{11}\right) \mathrm{d} x^{11}
$$

The $G$-field has a background value given by $[26,30,36,37]$

$$
\langle G\rangle=n_{i} \omega^{i(2,2)} \epsilon\left(x^{11}\right),
$$

where numbers $n_{i}$ are defined in $(2.14)$ and $\omega^{i(2,2)}\left(i=1, \ldots, h_{(2,2)}=h_{(1,1)}\right)$ are harmonic $(2,2)$ forms on $\mathcal{X}^{6}$ which satisfy the condition $\int_{\mathcal{X}^{6}} \omega_{i}^{(1,1)} \wedge \omega^{j(2,2)}=\delta_{i}^{j}$.

\footnotetext{
${ }^{5}$ The normalization convention for $G$ and $C$ is adopted from [31], and differs from that in [25] by factor $\ell_{11}^{3} / \sqrt{2}$ and $\ell_{11}^{3} / 6 \sqrt{2}$, respectively.
} 
In order to find the 4D effective axion we first construct a $5 \mathrm{D}$ effective theory by dimensional reduction of the 11D action (2.15). For this purpose we perform the following steps: We keep in $C$ and $G$ all relevant terms i.e. their background values and light $5 \mathrm{D}$ components:

$$
\begin{aligned}
& C=\langle C\rangle+\omega_{i}^{(1,1)} \wedge \mathcal{A}^{i}\left(x^{\alpha}\right)+C^{(5)}\left(x^{\alpha}\right)+\ldots, \\
& G=\langle G\rangle+\mathrm{d}\left(\omega_{i}^{(1,1)} \wedge \mathcal{A}^{i}\left(x^{\alpha}\right)\right)+G^{(5)}\left(x^{\alpha}\right)+\ldots,
\end{aligned}
$$

where 1-form fields $\mathcal{A}^{i}(x)$ and tensors $C^{(5)}$ and $G^{(5)}$ are 5D fields (have all indices tangent to $5 \mathrm{D}$ space and are functions of $5 \mathrm{D}$ coordinates) while $\langle C\rangle$ may be obtained from eqs. (2.19)(2.21). We substitute such $C$ and $G$ into the 11D action (2.15) and add to it a term with a 7 -form Lagrange multiplier to implement the modified Bianchi identity (2.16). Then, we integrate over the CY space. Finally, eliminating first the tensor fields $C^{(5)}$ and $G^{(5)}$ (using their equations of motion), we get the following relevant part of the $5 \mathrm{D}$ action

$$
\begin{aligned}
\mathcal{S}_{5, \text { axion }}= & -2 \pi \int_{\mathcal{M}^{5}}\left[\frac{\ell_{11}^{3}}{2 V_{X}}\left(\mathrm{~d} a+n_{i} \mathcal{A}^{i}\right) \wedge \star\left(\mathrm{d} a+n_{i} \mathcal{A}^{i}\right)+\frac{1}{2 \ell_{11}^{3}} G_{i j} \mathrm{~d} \mathcal{A}^{i} \wedge \star \mathrm{d} \mathcal{A}^{j}\right] \\
& +\int_{\mathcal{M}^{5}} \frac{1}{4 \pi} a\left[\left(\operatorname{tr} F_{(1)} \wedge F_{(1)}\right) \delta\left(x^{11}\right)+\left(\operatorname{tr} F_{(2)} \wedge F_{(2)}\right) \delta\left(x^{11}-\pi r_{11}\right)\right] \wedge \mathrm{d} x^{11},
\end{aligned}
$$

where kinetic function $G_{i j}$ was defined in (2.13) and $a$ is a scalar related to the field dual to $G^{(5)}$ by the relation

$$
\star_{5} G^{(5)}=-\frac{\ell_{11}^{3}}{V_{X}}\left(\mathrm{~d} a+n_{i} \mathcal{A}^{i}\right) .
$$

The scalar $a$ couples the same way to both $E_{8}$ gauge groups in analogy to the MI axion in (2.11). It is a priori not obvious whether the action (2.24) describes any more axions. The only fields present in (2.24) (in addition to the scalar $a$ and the gauge fields strengths $\left.F_{(j)}\right)$ are $h_{(1,1)} 5 \mathrm{D}$ vectors $\mathcal{A}^{i}$. We will see later that the components $\mathcal{A}_{11}^{i}$, which are $4 \mathrm{D}$ scalars, will correspond to $4 \mathrm{D}$ axions. The first line in (2.24) shows that they necessarily mix with the scalar $a$. Thus, the mixing among these fields must be taken into account in order to identify precisely all axions in this case. Such mixing will be discussed in the next section.

The action (2.24) is somewhat similar to the action (2.11) describing the MI axion in the weakly coupled case. Here, however, the definition of the MI axion appears to be ambiguous. Contrary to the weakly coupled string case, dualization of the tensor field strength involves also other axion candidates $\mathcal{A}_{11}^{i}$ with quantities depending on compactification details - numbers $n_{i}$ defined in (2.14) appear in relation (2.25). However, comparing the weakly and strongly coupled heterotic theories, we notice that axion fields have similar origin in both cases. The field $a$ originates from dualization of the tensor field strength ( $H$ in heterotic string theory and $G$ in heterotic M-theory) with no indices tangent to the CY space $\mathcal{X}^{6}$. The remaining axion fields are (parts of) coefficients in the expansion of the tensor field ( $B$ in heterotic string theory and $C$ in heterotic M-theory) in terms of $h_{(1,1)}$ harmonic $(1,1)$-forms on $\mathcal{X}^{6}$. Their number and properties do depend on the details 
of the compactification. Thus, one could call them MD-like axions and refer to $a$ as the MI-like one. ${ }^{6}$

There are two differences between the weakly and strongly coupled theories due to the fact that in the latter case compactification on $\mathcal{X}^{6}$ leads to a $5 \mathrm{D}$ effective theory. One of them consists in different transformation properties of MD axion fields. In the strongly coupled case they are components of $5 \mathrm{D}$ vectors and become scalars only after further compactification to 4D (field $a$ is a scalar already in 5D). The second difference is quite obvious: all the relevant fields in the M-theory case depend on 5D coordinates and only (some of) their $4 \mathrm{D}$ zero modes play the role of $4 \mathrm{D}$ axions.

\section{$3 \quad$ Mixing}

\subsection{Weakly coupled heterotic string}

In the $4 \mathrm{D}$ supergravity language axions are imaginary (pseudoscalar) components of chiral superfields. The MI axion belongs to the dilaton filed $\mathcal{S}$ while the MD axions are parts of the moduli $\mathcal{T}^{i}$ (the overall modulus $\mathcal{T}$ in the case of $h_{(1,1)}=1$ ). While the axions are welldefined at the level of the classical action, they mix via quantum corrections connected to the Green-Schwarz anomaly cancellation terms. In the simple case of the standard embedding the observable sector gauge group is broken to $E_{6}$ and one obtains

$$
f_{6}=\mathcal{S}+\epsilon \mathcal{T}, \quad f_{8}=\mathcal{S}-\epsilon \mathcal{T},
$$

where $f_{6}\left(f_{8}\right)$ is the gauge kinetic function of the observable (hidden) $E_{6}\left(E_{8}\right)$ and $\epsilon$ is a constant (fixed by the anomaly) [38-40]. In the case of some simple compactification schemes (e.g. orbifolds) this mixing can be calculated in explicit one-loop calculations of threshold corrections to the gauge kinetic functions [41, 42].

The identification of the physical QCD-axion will have to take into account this mixing. We shall not discuss this here in detail as we shall see later that it is impossible to obtain an axion decay constant within the "axion window" in the weakly coupled heterotic string. Both decay constants $f_{6}$ and $f_{8}$ will be of order of the string scale and cannot be lowered in the desired way. This will be different in the strongly coupled case.

\section{$3.2 \quad$ Heterotic M-theory}

As explained in the previous section, the axions in the heterotic M-theory originate from the $4 \mathrm{D}$ zero modes of the $5 \mathrm{D}$ fields $a$ (MI-like) and $\mathcal{A}_{11}^{i}$ (MD-like). We want to determine the mixing among them. However, it is obvious from (2.24) that these fields mix also with the remaining components of the $5 \mathrm{D}$ vectors $\mathcal{A}^{i}$. Thus, we have to eliminate this mixing first and this can be done by a choice of the gauge. The kinetic term for $\mathcal{A}^{i}$ present in $(2.24)$ (obtained from that of the $G$ field strength in the 11D action) depends on compactification details because the gauge kinetic function $G_{i j}$, defined in (2.13), is related to properties of

\footnotetext{
${ }^{6}$ The field $a$ corresponds to $C_{\mu \nu, 11}$ (while $C_{\mu \nu \rho}$ is responsible for the derivative of $a$ along the 11th dimension) in the strongly coupled case and to $B_{\mu \nu}$ in the weakly coupled one. The candidates for the remaining axions originate from $C_{m n, 11}$ and $B_{m n}$, respectively.
} 
$(1,1)$-forms on the internal CY space $\mathcal{X}^{6}$. In this section we consider the simplest case ${ }^{7}$ when there is only one such form (i.e. $h_{(1,1)}=1$ ) so there is only one gauge field $\mathcal{A} \equiv \mathcal{A}^{1}$. In this case, it turns out that

$$
G_{11}=3 V_{X}^{1 / 3}
$$

The mixing between $\mathcal{A}_{\mu}$ and $\left(\mathcal{A}_{11}, a\right)$ may be eliminated by choosing an appropriate gauge i.e. by adding the following gauge fixing term [24]

$$
S_{\text {g.f. }}=-\int_{\mathcal{M}^{5}} \frac{\sqrt{-g}}{2 g_{\mathcal{A}}^{2}} \hat{V}_{X}^{1 / 3}\left[g^{\alpha \beta} \partial_{\alpha} \mathcal{A}_{\beta}+g^{11,11} \frac{\partial_{11} \chi}{\chi} \mathcal{A}_{11}+n_{1} \hat{V}_{X}^{-4 / 3} g_{\mathcal{A}}^{2} F_{a}^{3} a\right]^{2},
$$

where

$$
\begin{aligned}
g_{\mathcal{A}}^{2} & =\frac{\ell_{11}^{3}}{6 \pi V_{X, 0}^{1 / 3}} \\
F_{a}^{3} & \equiv \frac{2 \pi \ell_{11}^{3}}{V_{X, 0}}, \\
\chi & \equiv \sqrt{-g} g^{11,11} g^{\mu \nu} \frac{\eta_{\mu \nu}}{4} \hat{V}_{X}^{1 / 3},
\end{aligned}
$$

and $\hat{V}_{X} \equiv V_{X} / V_{X, 0}$ with $V_{X, 0}$ being the CY volume at our boundary $x^{11}=0$ (convention for indices as in footnote 4 ). The 11D space-time is warped, so the background metric $g_{\alpha \beta}$ and the volume $V_{X}$ depend in general on the 11-th coordinate $x^{11}$. Thus, the gauge choice depends on the background geometry. In our previous work [25] we have shown that the $5 \mathrm{D}$ background geometry is given by

$$
\mathrm{d} s^{2}=\hat{V}_{X}^{-2 / 3}\left(e^{2 k_{1} x^{11}} \eta_{\mu \nu} \mathrm{d} x^{\mu} \mathrm{d} x^{\nu}+e^{2 k_{2} x^{11}}\left(\mathrm{~d} x^{11}\right)^{2}\right) .
$$

The prefactor $\hat{V}_{X}^{-2 / 3}$ is introduced to obtain the Einstein frame in $5 \mathrm{D}$. The exponents $k_{1}$ and $k_{2}$ may be written as

$$
k_{1}=-\hat{b} n M_{11}, \quad k_{2}=\hat{c}^{2} k_{1},
$$

where parameters $\hat{b}$ and $\hat{c}^{2}>0$ are determined by properties of the Kähler moduli, and $n$ is given by

$$
n=-\frac{1}{V_{X, 0}^{1 / 3}} \int_{\mathcal{X}^{6}} \omega \wedge \frac{1}{16 \pi^{2}}\left(\operatorname{tr} F_{(1)} \wedge F_{(1)}-\frac{1}{2} \operatorname{tr} \mathcal{R} \wedge \mathcal{R}\right),
$$

with the Kähler form $\omega$ of $\mathcal{X}^{6}$. The sign of this number is important because it determines the direction of warping along the 11-th dimension. It is positive (negative $k_{1}$ and $k_{2}$ ) for the standard embedding while it can be negative (postive $k_{1}$ and $k_{2}$ ) for non-standard embeddings. Only the latter case is of interest for our discussion as it will give us the possibility of lowering the axion scale significantly. For the present case of $h_{(1,1)}=1$ the above parameters read

$$
\hat{b}=\frac{\alpha_{\mathrm{GUT}}^{2 / 3}}{6(4 \pi)^{1 / 9}}, \quad \hat{c}^{2}=6,
$$

\footnotetext{
${ }^{7}$ In appendix A, we will work out a next simple example with $h_{(1,1)}=2$. We expect that essential features should not be much different for bigger values of $h_{(1,1)}$.
} 
and $n=n_{1}$ as defined in (2.14) with $V_{X} \rightarrow V_{X, 0}$. The volume modulus $\hat{V}_{X}$ is related to the dilaton $S$ of the General Linear Dilaton (GLD) model $[24,25]$ which has the "linear dilaton" background:

$$
\hat{V}_{X}=e^{\sqrt{2} S}=e^{k_{2} y} .
$$

After the gauge fixing (3.3), $\mathcal{A}_{\mu}$ is decoupled from $\mathcal{A}_{11}$ and $a$, and we are left with mixing between $\mathcal{A}_{11}$ and $a$ only. Diagonalizing it further, we obtain our final form for the $5 \mathrm{D}$ axion lagrangian:

$$
\begin{aligned}
S_{5, \text { axion }}=-\int_{\mathcal{M}^{5}} \mathrm{~d}^{5} x & {\left[\frac{1}{2} \eta^{\mu \nu} \partial_{\mu} \phi_{L} \partial_{\nu} \phi_{L}+\frac{1}{2} \eta^{\mu \nu} \partial_{\mu} \phi_{R} \partial_{\nu} \phi_{R}\right.} \\
& +\frac{1}{2} e^{2\left(k_{1}-k_{2}\right) x^{11}}\left[\left(\partial_{11} \phi_{L}+m_{\mathcal{A}} \phi_{L}\right)^{2}+\left(\partial_{11} \phi_{R}-m_{\mathcal{A}} \phi_{R}\right)^{2}\right] \\
& \left.+\frac{1}{4 \pi} a\left(\operatorname{tr} F_{(1)} \widetilde{F}_{(1)} \delta\left(x^{11}\right)+\operatorname{tr} F_{(2)} \widetilde{F}_{(2)} \delta\left(x^{11}-\pi r_{11}\right)\right)\right],
\end{aligned}
$$

where

$$
m_{\mathcal{A}}=\sqrt{\left(\frac{1}{2} k_{2}-k_{1}\right)^{2}+n_{1}^{2} g_{\mathcal{A}}^{2} F_{a}^{3}}=\frac{1}{2} k_{2}+k_{1} .
$$

Note that, in this notation, only the MI-like field $a$ appears to couple to the gauge fields $F_{(j)} \widetilde{F}_{(j)}$. The coupling of the MD-like axions is hidden in the mixing. The orthogonal axion fields $\phi_{L}$ and $\phi_{R}$ are expressed in terms of the axionic fields $a$ and $\mathcal{A}_{11}$ via the relations

$$
\begin{aligned}
& \phi_{L}=e^{-\left(\frac{1}{2} k_{2}-k_{1}\right) x^{11}}\left(a F_{a}^{3 / 2} \cos \beta-\mathcal{A}_{11} g_{\mathcal{A}}^{-1} \sin \beta\right), \\
& \phi_{R}=e^{-\left(\frac{1}{2} k_{2}-k_{1}\right) x^{11}}\left(-a F_{a}^{3 / 2} \sin \beta-\mathcal{A}_{11} g_{\mathcal{A}}^{-1} \cos \beta\right),
\end{aligned}
$$

with the mixing angle $\beta$ satisfying the condition

$$
\tan 2 \beta=-g_{\mathcal{A}} F_{a}^{3 / 2} \frac{n_{1}}{\frac{1}{2} k_{2}-k_{1}}=\frac{\sqrt{2 k_{1} k_{2}}}{\frac{1}{2} k_{2}-k_{1}} .
$$

For $\hat{c}^{2}=6\left(k_{2}=6 k_{1}\right)$, the mixing angle $\beta$ comes out as

$$
\beta=\frac{\pi}{6}
$$

The profiles of these axion fields in the 11-th dimension will be important for the discussion of possible candidates for a QCD-axion that solve the strong CP-problem on the observable brane. This will be explicitely discussed in the next section.

From (3.12), we see that the axions from heterotic M-theory are identified as "continuous clockwork axions" as explicitly discussed in $[22,24]$. The bulk gauge boson mass $m_{\mathcal{A}}$ serves as the clockwork parameter for the zero mode coupling hierarchy, while $k_{1}-k_{2} \equiv p$ controls the clockwork gear (KK mode) masses [24, 25]. An important difference from "discrete clockwork axion" $[16,17]$ is that $\phi_{L}$ and $\phi_{R}$ couple to $4 \mathrm{D}$ instantons only in the following combination:

$$
a=F_{a}^{-3 / 2} e^{\left(\frac{k_{2}}{2}-k_{1}\right) x^{11}}\left(\phi_{L} \cos \beta-\phi_{R} \sin \beta\right) .
$$


There is no such restriction in the discrete clockwork axion models. This is an example of the limitations of this continuous clockwork axion compared to the discrete models as pointed out in section 3.4 of $[24] .^{8}$

\section{Decay constants and couplings}

Let us now turn to a discussion of the scales of the axion decay constants.

\subsection{Weakly coupled heterotic string}

In the weakly coupled heterotic string the MI axion decay constant may be read off from the action (2.11) and equals

$$
f_{a}=\sqrt{\frac{2 \kappa_{10}^{2}}{V_{X} \ell_{s}^{4}}}=\frac{\alpha_{\mathrm{YM}}}{2 \sqrt{2} \pi} M_{\mathrm{P}},
$$

where $M_{\mathrm{P}}$ is the $4 \mathrm{D}$ reduced Planck mass and $\alpha_{\mathrm{YM}}$ is the $E_{8} \times E_{8}$ gauge coupling given by

$$
M_{\mathrm{P}}^{2}=\frac{V_{X}}{\kappa_{10}^{2}}, \quad \alpha_{\mathrm{YM}}^{-1}=\pi \alpha^{\prime} \frac{V_{X}}{\kappa_{10}^{2}} .
$$

The MD axions $b^{i}$ mix for general non-diagonal $G_{i j}$ and their decay constants depend on the details of the model. A typical value of such constants may be estimated as

$$
f_{b} \sim \sqrt{\frac{\ell_{s}^{4}}{8 \pi^{2} \kappa_{10}^{2}}} \sqrt{\frac{V_{X}}{V_{C}^{2}}}=\frac{1}{2 \pi f_{a} V_{C}} \gtrsim \frac{\alpha_{\mathrm{YM}}}{2 \sqrt{2} \pi} M_{\mathrm{P}},
$$

where $V_{C}$ measures an average volume of $(1,1)$ basis cycles $C_{i}$ on $\mathcal{X}^{6}$, and we have used $G_{i j} \sim V_{X} / V_{C}^{2}$ from (2.13) because $\int_{C_{i}} \omega_{i}=1$. The inequality derives from the upper bound $V_{C} \lesssim \alpha_{\mathrm{YM}}^{-1} \ell_{s}^{2}$ as argued in [31]. Therefore, both MI and MD axions have their decay constants around the GUT scale $\sim 10^{16} \mathrm{GeV}$. This is due to the fact that the string scale is tied to the $4 \mathrm{D}$ Planck scale by the relation $M_{\mathrm{P}}^{2}=\left(\pi \alpha_{\mathrm{YM}} \alpha^{\prime}\right)^{-1}$ as can be obtained from (4.2). Therefore we shall not be able to find a QCD-axion with a decay constant within the preferred axion window. In the strongly coupled heterotic M-theory the situation can be different. One might obtain axion decay constants much lower than the Planck scale as we are going to discuss in the next subsection.

\subsection{Heterotic M-theory}

Because of the clockwork parameter $m_{\mathcal{A}}$ present in (3.12), the $4 \mathrm{D}$ zero modes of $\phi_{L}$ and $\phi_{R}$ are exponentially localized towards the left boundary $\left(x^{11}=0\right)$ and right boundary $\left(x^{11}=\pi r_{11}\right)$, respectively. The $5 \mathrm{D}$ fields may be expanded in the $4 \mathrm{D}$ modes as

$$
\begin{aligned}
& \phi_{L}\left(x^{\mu}, x^{11}\right)=\sqrt{\frac{m_{\mathcal{A}}}{1-e^{-2 m_{\mathcal{A}} \pi r_{11}}}} e^{-m_{\mathcal{A}} x^{11}} a_{L}\left(x^{\mu}\right)+\sum_{n=1}^{\infty} \psi_{L}^{(n)}\left(x^{11}\right) \phi_{L}^{(n)}\left(x^{\mu}\right), \\
& \phi_{R}\left(x^{\mu}, x^{11}\right)=\sqrt{\frac{m_{\mathcal{A}}}{e^{2 m_{\mathcal{A}} \pi r_{11}}-1}} e^{m_{\mathcal{A}} x^{11}} a_{R}\left(x^{\mu}\right)+\sum_{n=1}^{\infty} \psi_{R}^{(n)}\left(x^{11}\right) \phi_{R}^{(n)}\left(x^{\mu}\right),
\end{aligned}
$$

\footnotetext{
${ }^{8}$ More specifically, none of these continuous clockwork axions can have a short periodic "wiggle" on top of its cosine potential due to the presence of the other axion.
} 
where $a_{L}\left(x^{\mu}\right)$ and $a_{R}\left(x^{\mu}\right)$ denote $4 \mathrm{D}$ zero modes which will play the role of axions (with the prefactors chosen to normalize their $4 \mathrm{D}$ kinetic terms canonically), while $\phi_{L, R}^{(n)}\left(x^{\mu}\right)$ are $4 \mathrm{D}$ massive KK modes (with profiles in the 11-th dimension given by $\psi_{L, R}^{(n)}\left(x^{11}\right)$ ).

As is obvious from (3.12), axions $a_{L}$ and $a_{R}$ do not mix via their kinetic terms but both couple to $\operatorname{tr} F \widetilde{F}$ at each brane, because $a$ contributes to $\phi_{L}$ and $\phi_{R}$ as shown in (3.14) and (3.15). The 4D Lagrangian for these axions has the form

$$
\begin{aligned}
\mathcal{L}_{4, \text { axion }}= & -\frac{1}{2}\left(\partial_{\mu} a_{L}\right)^{2}-\frac{1}{2}\left(\partial_{\mu} a_{R}\right)^{2} \\
& +\frac{1}{16 \pi^{2}}\left[\left(\frac{a_{L}}{f_{L 1}}-\frac{a_{R}}{f_{R 1}}\right) \operatorname{tr} F_{(1)} \widetilde{F}_{(1)}+\left(\frac{a_{L}}{f_{L 2}}-\frac{a_{R}}{f_{R 2}}\right) \operatorname{tr} F_{(2)} \widetilde{F}_{(2)}\right],
\end{aligned}
$$

where

$$
\begin{array}{ll}
f_{L 1}=\frac{f_{a}}{\cos \beta} \sqrt{1-\exp \left(-2 m_{\mathcal{A}} \pi r_{11}\right)}, & f_{L 2}=\frac{f_{a}}{\cos \beta} \frac{\sqrt{\exp \left(2 m_{\mathcal{A}} \pi r_{11}\right)-1}}{\exp \left(\left(\frac{1}{2} k_{2}-k_{1}\right) \pi r_{11}\right)} \\
f_{R 1}=\frac{f_{a}}{\sin \beta} \sqrt{\exp \left(2 m_{\mathcal{A}} \pi r_{11}\right)-1}, & f_{R 2}=\frac{f_{a}}{\sin \beta} \frac{\sqrt{1-\exp \left(-2 m_{\mathcal{A}} \pi r_{11}\right)}}{\exp \left(\left(\frac{1}{2} k_{2}-k_{1}\right) \pi r_{11}\right)}
\end{array}
$$

and

$$
f_{a}=\frac{F_{a}^{3 / 2}}{4 \pi \sqrt{m_{\mathcal{A}}}}=\frac{\sqrt{3 \pi}}{4 \pi} \frac{\alpha_{\mathrm{YM}}^{1 / 6}}{\sqrt{\left|n_{1}\right|}} \frac{1}{\ell_{11}} \sim 0.1 M_{11} .
$$

The couplings of a given axion to two branes are very different for large values of $\pi r_{11}$ due to the clockwork mechanism:

$$
\begin{aligned}
& \frac{f_{L 1}}{f_{L 2}}=\exp \left[-\left(m_{\mathcal{A}}-\left(\frac{1}{2} k_{2}-k_{1}\right)\right) \pi r_{11}\right], \\
& \frac{f_{R 2}}{f_{R 1}}=\exp \left[-\left(m_{\mathcal{A}}+\left(\frac{1}{2} k_{2}-k_{1}\right)\right) \pi r_{11}\right] .
\end{aligned}
$$

The coupling of the axion $a_{L}\left(a_{R}\right)$ to the left (right) brane at which it is exponentially localized is much bigger than its coupling to the right (left) brane.

The above exponential factors can be expressed in terms of the $4 \mathrm{D}$ Planck scale $M_{\mathrm{P}}$ and the $11 \mathrm{D}$ string scale $M_{11}$. According to [25],

$$
\frac{M_{\mathrm{P}}}{M_{11}} \simeq \gamma e^{k \pi r_{11}}
$$

where the factor $\gamma=\mathcal{O}(10 \div 100)$ depends on compactification while $k \equiv \frac{1}{2} k_{2}+k_{1}$ is the clockwork parameter controlling the coupling hierarchy for the zero mode graviton.

Notice that this clockwork parameter is the same as the one for axions, i.e. $m_{\mathcal{A}}=k$ as can be seen in (3.13). Therefore,

$$
\begin{aligned}
\exp \left(m_{\mathcal{A}} \pi r_{11}\right) & \simeq \frac{1}{\gamma} \frac{M_{\mathrm{P}}}{M_{11}}, \\
\exp \left(\left(\frac{1}{2} k_{2}-k_{1}\right) \pi r_{11}\right) & \simeq\left(\frac{1}{\gamma} \frac{M_{\mathrm{P}}}{M_{11}}\right)^{\left(\hat{c}^{2}-2\right) /\left(\hat{c}^{2}+2\right)},
\end{aligned}
$$


where the exponent of the second term $\left(\hat{c}^{2}-2\right) /\left(\hat{c}^{2}+2\right)=1 / 2$ for $\hat{c}^{2}=6$. This implies

$$
\begin{aligned}
f_{L 1} & \sim 0.1 M_{11}, & f_{L 2} & \sim \frac{0.1}{\sqrt{\gamma}} \sqrt{\frac{M_{11}}{M_{\mathrm{P}}}} M_{\mathrm{P}}, \\
f_{R 1} & \sim \frac{0.1}{\gamma} M_{\mathrm{P}}, & f_{R 2} & \sim 0.1 \sqrt{\gamma} \sqrt{\frac{M_{11}}{M_{\mathrm{P}}}} M_{11} .
\end{aligned}
$$

We see that in the heterotic M-theory case we could obtain axion decay constants that are small compared to the Planck scale. This mechanism requires a rather large value of $\pi r_{11}$ and thus a small value of the scale $M_{11}$. In fact, the above equations apparently reveal several options. In the case of $f_{L 1}$, for example, we have to choose $M_{11}$ to be an order of magnitude bigger than the axion decay constant. The strongest suppression appears for $f_{R 2}$, where $M_{11}$ might even be $2 \div 3$ orders of magnitude above the axion window. We might thus hope to obtain acceptable QCD-axion candidates for sufficiently low values of $M_{11}$. In the next section we shall see that among these various candidates there is only one, unique solution to the strong CP-problem.

\section{Towards a QCD axion}

Let us finally turn to the search for a suitable QCD axion candidate. It should have a small decay constant and we thus assume throughout this section that $\pi r_{11}$ is sufficiently large. The axion should, of course, have a sizeable coupling to the observable brane (where QCD is located) and a potential that is flat enough to dynamically adjust $\theta_{\mathrm{QCD}}$ to zero. The candidate should be a suitable combination of $a_{L}$ and $a_{R}$, as defined above.

The fields $a_{L}$ and $a_{R}$ are both massless at the level of the action (3.12) but become massive when the instanton effects are taken into account. Then, as we will see below, in general they are not mass eigenstates. The potential for the axions generated by instantons has the form

$$
\begin{aligned}
V\left(a_{L}, a_{R}\right)= & -\Lambda_{\mathrm{QCD}}^{4} \cos \left(\frac{a_{L}}{f_{L 1}}-\frac{a_{R}}{f_{R 1}}+\theta_{\mathrm{QCD}}\right)-\sum_{i} \Lambda_{(1) i}^{4} \cos \left(\frac{a_{L}}{f_{L 1}}-\frac{a_{R}}{f_{R 1}}+\theta_{(1) i}\right) \\
& -\sum_{j} \Lambda_{(2) j}^{4} \cos \left(\frac{a_{L}}{f_{L 2}}-\frac{a_{R}}{f_{R 2}}+\theta_{(2) j}\right),
\end{aligned}
$$

where $\Lambda_{(1) i}^{4}$ and $\Lambda_{(2) j}^{4}$ are terms induced by instantons other than QCD from the visible sector and hidden sector, respectively. The conditions for the minimum of this potential are:

$$
\begin{aligned}
\Lambda_{\mathrm{QCD}}^{4} \sin \left(\frac{a_{L}}{f_{L 1}}-\frac{a_{R}}{f_{R 1}}+\theta_{\mathrm{QCD}}\right)+\sum_{i} \Lambda_{(1) i}^{4} \sin \left(\frac{a_{L}}{f_{L 1}}-\frac{a_{R}}{f_{R 1}}+\theta_{(1) i}\right) & =0, \\
\sum_{j} \Lambda_{(2) j}^{4} \sin \left(\frac{a_{L}}{f_{L 2}}-\frac{a_{R}}{f_{R 2}}+\theta_{(2) j}\right) & =0 .
\end{aligned}
$$

In order to solve the strong CP problem, the visible sector instantons other than the QCD contribution must be suppressed,

$$
\Lambda_{(1) i}^{4} \lesssim 10^{-10} \Lambda_{\mathrm{QCD}}^{4}
$$


so in the following we set $\Lambda_{(1) i}=0$. The contributions to the potential from the hidden sector instantons, $\Lambda_{(2) j}^{4}$, may be sizable. For any values of $\Lambda_{\mathrm{QCD}}$ and $\Lambda_{(2) j}$ the fields $a_{L}$ and $a_{R}$ acquire such vacuum expectation values that both minimalization conditions (5.2) and (5.3) are fulfilled and $\theta_{\mathrm{QCD}}^{\mathrm{eff}}=0$.

The second derivatives of the potential (5.1) give the mass squared matrix for the axions

$$
\left(m^{2}\right)_{J K}=\frac{\Lambda_{\mathrm{QCD}}^{4}}{f_{J 1} f_{K 1}}+\frac{\bar{\Lambda}^{4}}{f_{J 2} f_{K 2}},
$$

where $J, K=L, R$ and $\bar{\Lambda}$ is defined by

$$
\bar{\Lambda}^{4}=\left.\sum_{j} \Lambda_{(2) j}^{4} \cos \left(\frac{a_{L}}{f_{L 2}}-\frac{a_{R}}{f_{R 2}}+\theta_{(2) j}\right)\right|_{\sum_{j} \Lambda_{(2) j}^{4} \sin \left(\frac{a_{L}}{f_{L 2}}-\frac{a_{R}}{f_{R 2}}+\theta_{(2) j}\right)=0} .
$$

The mass squared matrix (5.5) has two eigenvalues

$$
m_{a_{h}, a_{l}}^{2}=\frac{m_{L L}^{2}+m_{R R}^{2}}{2} \pm \frac{m_{L L}^{2}-m_{R R}^{2}}{2} \sqrt{1+\frac{2 m_{L R}^{2}}{m_{L L}^{2}-m_{R R}^{2}}},
$$

corresponding to the eigenvectors

$$
\left(\begin{array}{l}
a_{h} \\
a_{l}
\end{array}\right)=\left(\begin{array}{cc}
\cos \beta^{\prime} & \sin \beta^{\prime} \\
-\sin \beta^{\prime} & \cos \beta^{\prime}
\end{array}\right)\left(\begin{array}{l}
a_{L} \\
a_{R}
\end{array}\right) .
$$

The rotation angle $\beta^{\prime}$ fulfills the condition

$$
\tan 2 \beta^{\prime}=\frac{2 m_{L R}^{2}}{m_{L L}^{2}-m_{R R}^{2}}
$$

The axion action written in terms of the mass eigenstates has form analogous to (4.6):

$$
\begin{aligned}
\mathcal{L}_{4, \text { axion }}= & -\frac{1}{2}\left(\partial_{\mu} a_{h}\right)^{2}-\frac{1}{2}\left(\partial_{\mu} a_{l}\right)^{2} \\
& +\frac{1}{16 \pi^{2}}\left[\left(\frac{a_{h}}{f_{h 1}}-\frac{a_{l}}{f_{l 1}}\right) \operatorname{tr} F_{(1)} \widetilde{F}_{(1)}+\left(\frac{a_{h}}{f_{h 2}}-\frac{a_{l}}{f_{l 2}}\right) \operatorname{tr} F_{(2)} \widetilde{F}_{(2)}\right]
\end{aligned}
$$

where constants $f_{h i}$ and $f_{l i}$ are related to $f_{L i}$ and $f_{R i}$ by appropriate rotations by angle $\beta^{\prime}$.

In general, both mass eigenstate axions, $a_{h}$ and $a_{l}$, couple to both branes and so both could contribute to the solution of the strong CP problem. However, the situation simplifies considerably in the case of interest to us where a large $\pi r_{11}$ leads to $M_{11} \ll M_{\mathrm{P}}$. Such strong inequality is necessary, as follows from eqs. (4.15) and (4.16), to get any axion decay constants substantially smaller than the Planck scale, e.g. in the axion window (and helps also to address the hierarchy problem). The parameters $\Lambda_{(2) j}^{4}$ may be written as

$$
\Lambda_{(2) j}^{4}=\Lambda_{\mathrm{c}(2) j}^{4} e^{-8 \pi^{2} / g^{2}} \sim \Lambda_{\mathrm{c}(2) j}^{4} e^{-16 \pi^{2} \hat{V}}
$$


where $\Lambda_{\mathrm{c}(2) j}$ is the confinement scale of the gauge field $F_{(2) j}$. The CY volume modulus $\hat{V}$ at the second boundary may be estimated as

$$
\hat{V}=e^{k_{2} \pi r_{11}} \sim\left(\frac{M_{\mathrm{P}}}{M_{11}}\right)^{3 / 2} .
$$

Therefore, for $M_{11} \ll M_{\mathrm{P}}$, parameters $\Lambda_{(2) j}$ and their combination $\bar{\Lambda}^{4}$ defined in (5.6) will be typically very small. In the limit $\bar{\Lambda}^{4} \ll \Lambda_{\mathrm{QCD}}^{4}$ one may neglect the contribution from $\bar{\Lambda}^{4}$ to the axion mass matrix given by the second term on the r.h.s. of (5.5). Then, $m_{a_{h}}^{2} \approx \Lambda_{\mathrm{QCD}}^{4} \sqrt{f_{L 1}^{-2}+f_{R 1}^{-2}} \gg m_{a_{l}}^{2}$ and the expression for the rotation angle $\beta^{\prime}$ simplifies to $\tan \beta^{\prime}=f_{L 1} / f_{R 1}$. The couplings of the axions in this limit read

$$
\begin{aligned}
\frac{1}{f_{h 1}} & =\sqrt{\frac{1}{f_{L 1}^{2}}+\frac{1}{f_{R 1}^{2}}}, & \frac{1}{f_{h 2}} & =\frac{1}{\sqrt{f_{L 1}^{2}+f_{R 1}^{2}}}\left(\frac{f_{R 1}}{f_{L 2}}+\frac{f_{L 1}}{f_{R 2}}\right), \\
\frac{1}{f_{l 1}} & \sim 0, & \frac{1}{f_{l 2}} & =\frac{1}{\sqrt{f_{L 1}^{2}+f_{R 1}^{2}}}\left(\frac{f_{R 1}}{f_{R 2}}-\frac{f_{L 1}}{f_{L 2}}\right) .
\end{aligned}
$$

The lighter axion, $a_{l}$, essentially decouples from QCD. The heavier axion, $a_{h}$, solves the strong CP problem but couples also to the hidden brane $\left(f_{h 2} \neq \infty\right)$. From (4.15) and (4.16), the decay constants are estimated as

$$
f_{h 1} \sim 0.1 M_{11}, \quad f_{h 2} \sim 0.1 \gamma^{-1 / 2} \sqrt{M_{\mathrm{P}} M_{11}}, \quad f_{l 2} \sim 0.1 \gamma^{1 / 2} M_{11} \sqrt{\frac{M_{11}}{M_{\mathrm{P}}}}
$$

Thus our QCD axion $a_{h}$ has the decay constant about one order of magnitude below the string scale $f_{h 1} \sim 0.1 M_{11}$. So, if the string scale $M_{11}$ lies in the "axion window" moved up by one order of magnitude,

$$
10^{10} \mathrm{GeV} \lesssim M_{11} \lesssim 10^{13} \mathrm{GeV}
$$

a phenomenologically acceptable solution to the strong CP problem is realized. In our previous work [25], we showed that the string scale $M_{11}$ can be lowered down to the $\mathrm{TeV}$ scale through a large warped 11-th dimension without violating current experimental bounds on the size of extra dimension. So it is not difficult to have the string scale lying in or slightly above the axion window as a result of the warping.

The relevant candidate for the QCD-axion is thus $a_{h}$. There is a second axion $a_{l}$ whose decay constant $f_{l 2}$ is parametrically even smaller than $M_{11}$. However, it cannot play the role of a QCD-axion as it essentially decouples from the observable sector. This observation extends and completes previous discussions of axions embedded in string theory [31].

The second axionic state $a_{l}$ couples predominantly to the hidden sector. In the case of large $r_{11}$ under consideration, its mass will be extremely small as the contributions of the hidden sector instantons are tiny. The appearance of the ultra-light axion is a direct consequence of the presence of the QCD-axion $a_{h}$ with decay constant compatible with the "axion window". The mass of the ULA, its phenomenological properties and possible cosmological implications [43] are strongly model dependent and should be subject to future investigations. 


\section{Conclusions and outlook}

In the present paper we have applied the previously discussed clockwork mechanism within the framework of heterotic M-theory [25] to axionic fields that could serve as candidates for a solution to the strong CP-problem. The decay constant $f_{a}$ of the corresponding axion field should be inside the "axion window" around $10^{11} \mathrm{GeV}$. Although there is a variety of axion fields in the 10D heterotic string theory, it is impossible to fulfill this requirement. In 11D heterotic M-theory, however, this is different. We can lower the axion decay constants under the same conditions and for the same reasons as we lowered the weak scale to the TeV-scale in ref. [25]. The typical set-up is a warped $7 \mathrm{D}$ compactification as the semi-direct product of an interval (with length $\pi r_{11}$ ) and a $6 \mathrm{D}$ Calabi-Yau manifold. A lower value of $f_{a}$ requires a rather large value of $r_{11}$ and a smaller value of $M_{11}$ (the fundamental mass scale of M-theory). At an intermediate step in this compactification, the candidate 4D axion fields will reside in 5D supergravity multiplets. In a hypermultiplet the 4D axion derives from a 5D scalar field, whereas in a gravity-multiplet and a vector-multiplet (relevant if $\left.h_{(1,1)}>1\right)$ it is a component of a $5 \mathrm{D}$ vector field. Generically, the $4 \mathrm{D}$ axion candidate will be a linear combination of these multiplets. As a prototype model we consider a CalabiYau manifold with $h_{(1,1)}=1$ and thus a two-axion system with one of these fields each. The case for $h_{(1,1)}>1$ is qualitatively similar and is discussed in appendix A.

A priori we can find various values for the axion decay constants under consideration (see e.g. formulae (4.15)-(4.16)). Low values of $f_{a}$ are possible in the case of large $r_{11}$ and small $M_{11}$. If we concentrate on an axion that could solve the strong CP-problem, however, only one of these choices is possible: the one with the string scale one order of magnitude above the axion decay constant: $M_{11} \sim 10 f_{a}$. This QCD-axion is accompanied by a second axion that is essentially decoupled form the observable sector gauge groups. Due to a type of "see-saw" mechanism, this second axion is extremely light (due to the suppression of the hidden sector instanton contributions). The decay constant of the ultra-light axion (ULA) is smaller than that of the QCD-axion (as $f_{l 2}$ in formula (5.15)). The exact value of the mass and the phenomenological properties of the ULA are strongly model dependent (especially in the case $h_{(1,1)}>1$ ). Although the ULA decouples from the observable sector and will be difficult to detect in particle physics experiments, the stronger coupling to the hidden sector might eventually be of cosmological relevance. This should be subject to future investigations.

\section{Acknowledgments}

SHI acknowledges partial support from Basic Science Research Program through the National Research Foundation of Korea (NRF) funded by the Ministry of Education (NRF2017R1D1A1B06033701 and 2019R1I1A1A01060680) and the Korea government (MSIP) (NRF-2018R1C1B3001379). SHI also thanks the CERN-Korea TH Institute, where valuable comments were given to this work. HPN thanks the CERN Theory Department for hospitality and support. MO acknowledges partial support from National Science Centre, Poland, grants DEC-2015/18/M/ST2/00054 and DEC-2016/23/G/ST2/04301. 


\section{A Axion couplings for $h_{(1,1)}=2$}

In this appendix, we provide more detailed formulae for the axion lagrangian and work out a simple example for $h_{(1,1)}>1$. The $5 \mathrm{D}$ axion effective lagrangian is given in (2.24):

$$
\begin{aligned}
\mathcal{S}_{5, \text { axion }}= & -2 \pi \int_{\mathcal{M}^{5}}\left[\frac{\ell_{11}^{3}}{2 V_{X}}\left(\mathrm{~d} a+n_{i} \mathcal{A}^{i}\right) \wedge \star\left(\mathrm{d} a+n_{i} \mathcal{A}^{i}\right)+\frac{1}{2 \ell_{11}^{3}} G_{i j} \mathrm{~d} \mathcal{A}^{i} \wedge \star \mathrm{d} \mathcal{A}^{j}\right] \\
& +\int_{\mathcal{M}^{5}} \frac{1}{4 \pi} a\left[\left(\operatorname{tr} F_{(1)} \wedge F_{(1)}\right) \delta\left(x^{11}\right)+\left(\operatorname{tr} F_{(2)} \wedge F_{(2)}\right) \delta\left(x^{11}-\pi r_{11}\right)\right] \wedge \mathrm{d} x^{11}
\end{aligned}
$$

The kinetic matrix $G_{i j}$ for the gauge fields is given by (2.13):

$$
\begin{aligned}
G_{i j} & =\int_{\mathcal{X}^{6}} \omega_{i}^{(1,1)} \wedge \star \omega_{j}^{(1,1)} \\
& =-V_{X}^{1 / 3}\left[d_{i j k} X^{k}-\frac{1}{4}\left(d_{i l m} X^{l} X^{m}\right)\left(d_{j n p} X^{n} X^{p}\right)\right]
\end{aligned}
$$

where the indices run over $i, j, k, \ldots=1, \ldots, h_{(1,1)}$ and

$$
d_{i j k} \equiv \int_{\mathcal{X}^{6}} \omega_{i}^{(1,1)} \wedge \omega_{j}^{(1,1)} \wedge \omega_{k}^{(1,1)}
$$

are the CY intersection numbers, and the Kähler moduli $X^{i}$ satisfy the constraint

$$
\frac{1}{6} d_{i j k} X^{i} X^{j} X^{k}=1
$$

In the case of $h_{(1,1)}=1, d_{111}=6$ and $X^{1}=1$ so that $G_{11}=3 V_{X}^{1 / 3}$ as used in (3.2).

As a simple example with $h_{(1,1)}>1$, let us consider $h_{(1,1)}=2$ and the CY intersection number $d_{112} \neq 0$ while the other $d_{i j k}$ vanish. This example was also considered in [25]. In this case, the constraint (A.4) becomes

$$
d_{112}\left(X^{1}\right)^{2} X^{2}=2
$$

which may be solved by

$$
\begin{aligned}
X^{1} & =\frac{1}{\eta} e^{-b S_{1}}, \\
X^{2} & =\frac{1}{\eta} e^{2 b S_{1}}
\end{aligned}
$$

where $\eta=\left(d_{112} / 2\right)^{1 / 3}$ and $b=1 / \sqrt{3}$ to canonically normalize $S_{1}$. On the other hand, the kinetic matrix (A.2) comes out as

$$
\begin{aligned}
& G_{11}=2 V_{X}^{1 / 3}\left(X^{1}\right)^{-2} \\
& G_{22}=V_{X}^{1 / 3}\left(X^{2}\right)^{-2} \\
& G_{12}=0
\end{aligned}
$$


It was shown in [25] that there are three qualitatively different background solutions. In the first case, for which both flux numbers $n_{1}$ and $n_{2}$ are non-vanishing, ${ }^{9}$ the Kähler modulus $S_{1}$ is stabilized at $\sqrt{3} S_{1}=\ln \left(n_{1} / 2 n_{2}\right)$, and the background geometry is the same as for $h_{(1,1)}=1$, i.e. as given in (3.10) and (3.11). The gauge couplings for $\mathcal{A}_{1}$ and $\mathcal{A}_{2}$ are then

$$
\begin{aligned}
& g_{\mathcal{A}_{1}}^{2}=\frac{\ell_{11}^{3}}{6 \pi V_{X, 0}^{1 / 3}} \frac{3}{2 \eta^{2}}\left(\frac{2 n_{2}}{n_{1}}\right)^{2 / 3}, \\
& g_{\mathcal{A}_{2}}^{2}=\frac{\ell_{11}^{3}}{6 \pi V_{X, 0}^{1 / 3}} \frac{3}{\eta^{2}}\left(\frac{n_{1}}{2 n_{2}}\right)^{4 / 3} .
\end{aligned}
$$

Compared to $h_{(1,1)}=1$, we just have to make the following replacement in the gauge field basis where the gauge couplings do not appear in kinetic terms:

$$
\begin{aligned}
\mathcal{A} & \rightarrow \tilde{\mathcal{A}} \equiv \frac{n_{1} g_{\mathcal{A}_{1}} \mathcal{A}_{1}+n_{2} g_{\mathcal{A}_{2}} \mathcal{A}_{2}}{\sqrt{n_{1}^{2} g_{\mathcal{A}_{1}}^{2}+n_{2}^{2} g_{\mathcal{A}_{2}}^{2}}}, \\
n_{1} g_{\mathcal{A}} & \rightarrow \sqrt{n_{1}^{2} g_{\mathcal{A}_{1}}^{2}+n_{2}^{2} g_{\mathcal{A}_{2}}^{2}} .
\end{aligned}
$$

Only the above linear combination of the gauge fields mixes with $a$, while the orthogonal one does not. So the gauge field orthogonal to $\tilde{\mathcal{A}}$ is irrelevant for the axion lagrangian unless there is another source of axion mixing. ${ }^{10}$ Then it turns out that the clockwork effective lagrangian is equivalent to (3.12) with the same gauge boson mass $m_{\mathcal{A}}$, and the clockwork fields $\phi_{L}$ and $\phi_{R}$ are related to $a$ and $\tilde{\mathcal{A}}_{11}$ by the same relation (3.14)-(3.15) and mixing angle (3.16).

The two other cases are obtained when one of the flux numbers is vanishing, i.e. either $n_{1}=0$ or $n_{2}=0$. For these cases, obviously only one of the gauge fields $\left(\mathcal{A}_{2}\right.$ or $\mathcal{A}_{1}$, respectively) mixes with $a$, so the other gauge field does not participate in the axion lagrangian at this level. It was shown in [25] that for these cases the Kähler modulus $S_{1}$ is identified as a component of the GLD dilaton together with the volume modulus $S_{V}=$ $(1 / \sqrt{2}) \ln \hat{V}_{X}$. This renders the geometric parameters $\hat{b}$ and $\hat{c}^{2}$ in $(3.8)$ to be different from the $h_{(1,1)}=1$ case. In particular, $\hat{c}^{2}=k_{2} / k_{1}$, which is 6 in the case of $h_{(1,1)}=1$, becomes

$$
\hat{c}^{2}= \begin{cases}7 & \text { for } n_{1} \neq 0, n_{2}=0 \\ 10 & \text { for } n_{1}=0, n_{2} \neq 0\end{cases}
$$

\footnotetext{
${ }^{9}$ Here the flux number $n_{i}$ is related to the dimensionful flux factor $\mu_{i}$ defined in [25] by the following relation,

$$
n_{i}=-\frac{V_{X, 0}^{2 / 3}}{4 \sqrt{2} \ell_{11}^{3}} \mu_{i} .
$$

${ }^{10}$ Including membrane instanton effects (e.g. [44]), the additional axion candidate from the 11-th component of this orthogonal gauge field can mix with $\tilde{\mathcal{A}}_{11}$. However, this effect is strongly model-dependent and typically small as the membrane instanton amplitude is either comparable to or smaller than the hidden YM instanton amplitude (5.11).
} 
Furthermore, since $S_{1}$ has a $x^{11}$-dependent background value, the gauge fixing term (3.3) should be generalized by the replacement

$$
V_{X}^{1 / 3} \rightarrow V_{X}^{1 / 3} \exp \left(2\left(1-\frac{6}{\hat{c}^{2}}\right) k_{2} x^{11}\right)
$$

The resultant clockwork effective lagrangian still has the same form as (3.12). The gauge boson mass $m_{\mathcal{A}}$ turns out to be

$$
m_{\mathcal{A}}=\sqrt{\left(\frac{1}{2} k_{2}-k_{1}-\left(1-\frac{6}{\hat{c}^{2}}\right) k_{2}\right)^{2}+n_{i}^{2} g_{\mathcal{A}}^{2} F_{a}^{3}}=\frac{1}{2} k_{2}+k_{1},
$$

so it has the same form as (3.13). On the other hand, the clockwork fields $\phi_{L}$ and $\phi_{R}$ are related to $a$ and $\mathcal{A}_{11}$ via the generalized relations

$$
\begin{aligned}
& \phi_{L}=e^{-\left(\frac{1}{2} k_{2}-k_{1}-\left(1-\frac{6}{\hat{c}^{2}}\right) k_{2}\right) x^{11}}\left(a F_{a}^{3 / 2} \cos \beta-\mathcal{A}_{11} g_{\mathcal{A}}^{-1} \sin \beta\right), \\
& \phi_{R}=e^{-\left(\frac{1}{2} k_{2}-k_{1}-\left(1-\frac{6}{\hat{c}^{2}}\right) k_{2}\right) x^{11}}\left(-a F_{a}^{3 / 2} \sin \beta-\mathcal{A}_{11} g_{\mathcal{A}}^{-1} \cos \beta\right),
\end{aligned}
$$

with the mixing angle $\beta$ satisfying the condition

$$
\tan 2 \beta=-g_{\mathcal{A}} F_{a}^{3 / 2} \frac{n_{i}}{\frac{1}{2} k_{2}-k_{1}-\left(1-\frac{6}{\hat{c}^{2}}\right) k_{2}}=\frac{\sqrt{\left(2 k_{1}+\left(1-\frac{6}{\hat{c}^{2}}\right) k_{2}\right)\left(k_{2}-\left(1-\frac{6}{\hat{c}^{2}}\right) k_{2}\right)}}{\frac{1}{2} k_{2}-k_{1}-\left(1-\frac{6}{\hat{c}^{2}}\right) k_{2}} .
$$

\section{B Kaluza-Klein axions}

In this section we discuss properties of Kaluza-Klein axion excitations in heterotic Mtheory. Their spectrum and couplings can be obtained from the continuum clockwork lagrangian (3.12). Detailed calculations were carried out in [24]. The analysis indicates that both $n$-th KK states, $\phi_{L}^{(n)}$ and $\phi_{R}^{(n)}$ with $n=1,2, \ldots$, have the same mass which is approximately given by

$$
M_{n} \approx\left(n-\frac{1}{4}+\frac{m_{\mathcal{A}}}{2|p|}\right) \pi|p| \exp \left(-|p| \pi r_{11}\right)
$$

where $p \equiv k_{1}-k_{2}$, as defined in [25], controls the clockwork gear (KK mode) masses. In terms of the 11D Planck mass $M_{11}$ and $4 \mathrm{D}$ Planck mass $M_{\mathrm{P}}$, this is estimated as [25]

$$
M_{n} \sim 30 n M_{11}\left(\frac{M_{11}}{M_{\mathrm{P}}}\right)^{2\left(\hat{c}^{2}-1\right) /\left(\hat{c}^{2}+2\right)} .
$$

Applying the heterotic bound $\hat{c}^{2} \geq 6$ argued in [25], the lightest KK mode mass $M_{1}$ is parametrically smaller than $M_{11}$. However, heavy KK modes with very large $n$ can have masses comparable to $M_{11}$ for which the above approximate formula is still valid.

The couplings to the boundary fields may be written as

$$
\frac{1}{16 \pi^{2}}\left[\sum_{n=1}^{\infty}\left(\frac{\phi_{L}^{(n)}}{f_{L 1}^{(n)}}-\frac{\phi_{R}^{(n)}}{f_{R 1}^{(n)}}\right) \operatorname{tr} F_{(1)} \widetilde{F}_{(1)}+\sum_{n=1}^{\infty}\left(\frac{\phi_{L}^{(n)}}{f_{L 2}^{(n)}}-\frac{\phi_{R}^{(n)}}{f_{R 2}^{(n)}}\right) \operatorname{tr} F_{(2)} \widetilde{F}_{(2)}\right],
$$


where

$$
\begin{aligned}
f_{L 1}^{(n)} & \approx \frac{f_{a}}{\cos \beta} \sqrt{\frac{m_{\mathcal{A}}}{|p| \pi}} \Gamma\left(\frac{m_{\mathcal{A}}}{|p|}\right)\left[\frac{\pi}{2}\left(n-\frac{1}{4}+\frac{m_{\mathcal{A}}}{2|p|}\right)\right]^{-\frac{m_{\mathcal{A}}}{|p| \frac{1}{2}}} \exp \left(m_{\mathcal{A}} \pi r_{11}\right), \\
f_{L 2}^{(n)} & \approx(-1)^{n} \frac{f_{a}}{\cos \beta} \sqrt{\frac{m_{\mathcal{A}}}{|p| \pi}} \exp \left(-\left(\frac{1}{2} k_{2}-k_{1}\right) \pi r_{11}\right), \\
f_{R i}^{(n)} & \approx \cot \beta f_{L i}^{(n)},
\end{aligned}
$$

with $f_{a}$ defined in (4.9). This means

$$
\begin{aligned}
& f_{L 1}^{(n)} \sim f_{R 1}^{(n)} \sim M_{\mathrm{P}}\left(\frac{M_{1}}{M_{n}}\right)^{3 / 2\left(\hat{c}^{2}-1\right)}, \\
& f_{L 2}^{(n)} \sim f_{R 2}^{(n)} \sim M_{11}\left(\frac{M_{11}}{M_{\mathrm{P}}}\right)^{\left(\hat{c}^{2}-2\right) /\left(\hat{c}^{2}+2\right)} .
\end{aligned}
$$

We see that light KK modes with small $n$ couple to the visible sector with decay constants around the $4 \mathrm{D}$ Planck scale $M_{\mathrm{P}}$. Heavy KK modes with $M_{n} \sim M_{11}$ can have quite lower decay constants below $M_{\mathrm{P}}$ as pointed out in [25] as a characteristic property of the heterotic clockwork due to $\hat{c}^{2}>1$. On the other hand, the decay constants $f_{L 2}^{(n)}$ and $f_{R 2}^{(n)}$ to the hidden sector are parametrically smaller than $M_{11}$. Nevertheless, since the hidden sector gauge coupling is small when $M_{11}$ is below $M_{\mathrm{P}}$,

$$
g_{(2)}^{2} \sim \hat{V}_{X}^{-1} \sim\left(\frac{M_{11}}{M_{\mathrm{P}}}\right)^{12 /\left(\hat{c}^{2}+2\right)}
$$

the net coupling to the hidden sector is

$$
\frac{g_{(2)}^{2}}{f_{L 2}^{(n)}} \sim \frac{g_{(2)}^{2}}{f_{R 2}^{(n)}} \sim \frac{1}{M_{11}}\left(\frac{M_{11}}{M_{\mathrm{P}}}\right)^{\left(14-\hat{c}^{2}\right) /\left(\hat{c}^{2}+2\right)} .
$$

This is still around $1 / M_{\mathrm{P}}$ or quite smaller than $1 / M_{11}$ for the known examples $\hat{c}^{2}=6,7,10$ in $[25]$.

Let us focus on the simplest example $\hat{c}^{2}=6$ with $M_{11}$ lying around the axion window $\left(10^{10} \mathrm{GeV}<M_{11}<10^{13} \mathrm{GeV}\right)$ in order to solve the strong CP problem with the zero mode axion. The KK axion masses are then

$$
M_{n} \approx 9 n \mathrm{GeV}\left(\frac{M_{11}}{10^{10} \mathrm{GeV}}\right)^{9 / 4},
$$

while the KK axion decay constants are

$$
\begin{aligned}
f_{L 1}^{(n)} & \sim f_{R 1}^{(n)} \sim \frac{M_{\mathrm{P}}}{n^{3 / 10}}>2.4 \times 10^{15} \mathrm{GeV}\left(\frac{M_{11}}{10^{10} \mathrm{GeV}}\right)^{3 / 8}, \\
f_{L 2}^{(n)} / g_{(2)}^{2} & \sim f_{R 2}^{(n)} / g_{(2)}^{2} \sim M_{\mathrm{P}},
\end{aligned}
$$

where the net couplings including the gauge coupling are estimated for the hidden sector because the gauge coupling may be small. Therefore, if $M_{11}=10^{10} \mathrm{GeV}$ for instance, we 
have $\mathrm{KK}$ axions of $10 \mathrm{GeV}$ scale mass gap with decay constants ranging from the $4 \mathrm{D}$ Planck scale all the way down to about $10^{15} \mathrm{GeV}$. These are so-called axion-like particles (ALP) present in heterotic M-theory accompanying the QCD axion.

Typically the KK axions of masses below $10^{6} \mathrm{GeV}$ have long enough life time to cause a problem for the standard cosmology. A straightforward way to cure this problem is to have a Hubble scale during inflation lower than the lightest $\mathrm{KK}$ axion mass in order to suppress their production [45]. As can be seen in (B.11), the lightest KK axion mass $M_{1}$ for $M_{11}>10^{10} \mathrm{GeV}$ is well above $H_{\mathrm{inf}} \sim 0.1 \mathrm{GeV}$ for successful baryogenesis.

Open Access. This article is distributed under the terms of the Creative Commons Attribution License (CC-BY 4.0), which permits any use, distribution and reproduction in any medium, provided the original author(s) and source are credited.

\section{References}

[1] R.D. Peccei and H.R. Quinn, CP Conservation in the Presence of Instantons, Phys. Rev. Lett. 38 (1977) 1440 [INSPIRE].

[2] K. Freese, J.A. Frieman and A.V. Olinto, Natural inflation with pseudo-Nambu-Goldstone bosons, Phys. Rev. Lett. 65 (1990) 3233 [INSPIRE].

[3] J.E. Kim, Light Pseudoscalars, Particle Physics and Cosmology, Phys. Rept. 150 (1987) 1 [INSPIRE].

[4] P.J. Steinhardt and M.S. Turner, Saving the Invisible Axion, Phys. Lett. 129B (1983) 51 [INSPIRE].

[5] M. Kawasaki, T. Moroi and T. Yanagida, Can decaying particles raise the upper bound on the Peccei-Quinn scale?, Phys. Lett. B 383 (1996) 313 [hep-ph/9510461] [INSPIRE].

[6] G.R. Dvali, Removing the cosmological bound on the axion scale, hep-ph/9505253 [INSPIRE].

[7] T. Banks and M. Dine, The cosmology of string theoretic axions, Nucl. Phys. B 505 (1997) 445 [hep-th/9608197] [INSPIRE].

[8] R.T. Co, E. Gonzalez and K. Harigaya, Axion Misalignment Driven to the Bottom, JHEP 05 (2019) 162 [arXiv: 1812.11186] [INSPIRE].

[9] K. Freese and W.H. Kinney, Natural Inflation: Consistency with Cosmic Microwave Background Observations of Planck and BICEP2, JCAP 03 (2015) 044 [arXiv:1403.5277] [INSPIRE].

[10] D.J.E. Marsh, Axion Cosmology, Phys. Rept. 643 (2016) 1 [arXiv:1510.07633] [InSPIRE].

[11] J.E. Kim, H.P. Nilles and M. Peloso, Completing natural inflation, JCAP 01 (2005) 005 [hep-ph/0409138] [INSPIRE].

[12] R. Kappl, S. Krippendorf and H.P. Nilles, Aligned Natural Inflation: Monodromies of two Axions, Phys. Lett. B 737 (2014) 124 [arXiv:1404.7127] [InSPIRE].

[13] K. Choi, H. Kim and S. Yun, Natural inflation with multiple sub-Planckian axions, Phys. Rev. D 90 (2014) 023545 [arXiv: 1404.6209] [INSPIRE].

[14] T. Higaki, K.S. Jeong, N. Kitajima and F. Takahashi, The QCD Axion from Aligned Axions and Diphoton Excess, Phys. Lett. B 755 (2016) 13 [arXiv:1512.05295] [inSPIRE]. 
[15] P.W. Graham, D.E. Kaplan and S. Rajendran, Cosmological Relaxation of the Electroweak Scale, Phys. Rev. Lett. 115 (2015) 221801 [arXiv:1504.07551] [INSPIRE].

[16] K. Choi and S.H. Im, Realizing the relaxion from multiple axions and its UV completion with high scale supersymmetry, JHEP 01 (2016) 149 [arXiv:1511.00132] [INSPIRE].

[17] D.E. Kaplan and R. Rattazzi, Large field excursions and approximate discrete symmetries from a clockwork axion, Phys. Rev. D 93 (2016) 085007 [arXiv:1511.01827] [INSPIRE].

[18] K.-S. Choi, H.P. Nilles, S. Ramos-Sanchez and P.K.S. Vaudrevange, Accions, Phys. Lett. B 675 (2009) 381 [arXiv:0902.3070] [INSPIRE].

[19] N. Arkani-Hamed, A.G. Cohen and H. Georgi, (De)constructing dimensions, Phys. Rev. Lett. 86 (2001) 4757 [hep-th/0104005] [INSPIRE].

[20] C.T. Hill, S. Pokorski and J. Wang, Gauge Invariant Effective Lagrangian for Kaluza-Klein Modes, Phys. Rev. D 64 (2001) 105005 [hep-th/0104035] [INSPIRE].

[21] G.F. Giudice and M. McCullough, A Clockwork Theory, JHEP 02 (2017) 036 [arXiv: 1610.07962] [INSPIRE].

[22] N. Craig, I. Garcia Garcia and D. Sutherland, Disassembling the Clockwork Mechanism, JHEP 10 (2017) 018 [arXiv:1704.07831] [INSPIRE].

[23] G.F. Giudice and M. McCullough, Comment on "Disassembling the Clockwork Mechanism", arXiv:1705.10162 [INSPIRE].

[24] K. Choi, S.H. Im and C.S. Shin, General Continuum Clockwork, JHEP 07 (2018) 113 [arXiv: 1711.06228] [inSPIRE].

[25] S.H. Im, H.P. Nilles and M. Olechowski, Heterotic M-theory from the Clockwork Perspective, JHEP 01 (2019) 151 [arXiv: 1811.11838] [INSPIRE].

[26] P. Hořava and E. Witten, Eleven-dimensional supergravity on a manifold with boundary, Nucl. Phys. B 475 (1996) 94 [hep-th/9603142] [INSPIRE].

[27] L. Randall and R. Sundrum, A large mass hierarchy from a small extra dimension, Phys. Rev. Lett. 83 (1999) 3370 [hep-ph/9905221] [INSPIRE].

[28] N. Arkani-Hamed, S. Dimopoulos and G.R. Dvali, The hierarchy problem and new dimensions at a millimeter, Phys. Lett. B 429 (1998) 263 [hep-ph/9803315] [INSPIRE].

[29] I. Antoniadis, S. Dimopoulos and A. Giveon, Little string theory at a TeV, JHEP 05 (2001) 055 [hep-th/0103033] [INSPIRE].

[30] A. Lukas, B.A. Ovrut, K.S. Stelle and D. Waldram, Heterotic M-theory in five-dimensions, Nucl. Phys. B 552 (1999) 246 [hep-th/9806051] [INSPIRE].

[31] P. Svrček and E. Witten, Axions In String Theory, JHEP 06 (2006) 051 [hep-th/0605206] [INSPIRE].

[32] E. Witten, Some Properties of O(32) Superstrings, Phys. Lett. 149B (1984) 351 [INSPIRE].

[33] M.B. Green and J.H. Schwarz, Anomaly Cancellation in Supersymmetric D $=10$ Gauge Theory and Superstring Theory, Phys. Lett. 149B (1984) 117 [InSPIRE].

[34] S.M. Barr, Harmless Axions in Superstring Theories, Phys. Lett. 158B (1985) 397 [INSPIRE].

[35] J.O. Conrad, Brane tensions and coupling constants from within M-theory, Phys. Lett. B 421 (1998) 119 [hep-th/9708031] [INSPIRE]. 
[36] E. Witten, Strong coupling expansion of Calabi-Yau compactification, Nucl. Phys. B 471 (1996) 135 [hep-th/9602070] [INSPIRE].

[37] A. Lukas, B.A. Ovrut, K.S. Stelle and D. Waldram, The universe as a domain wall, Phys. Rev. D 59 (1999) 086001 [hep-th/9803235] [INSPIRE].

[38] J.P. Derendinger, L.E. Ibáñez and H.P. Nilles, On the Low-Energy Limit of Superstring Theories, Nucl. Phys. B 267 (1986) 365 [INSPIRE].

[39] K. Choi and J.E. Kim, Compactification and Axions in $E_{8} \times E_{8}$-prime Superstring Models, Phys. Lett. 165B (1985) 71 [INSPIRE].

[40] L.E. Ibáñez and H.P. Nilles, Low-Energy Remnants of Superstring Anomaly Cancellation Terms, Phys. Lett. 169B (1986) 354 [INSPIRE].

[41] L.J. Dixon, V. Kaplunovsky and J. Louis, Moduli dependence of string loop corrections to gauge coupling constants, Nucl. Phys. B 355 (1991) 649 [INSPIRE].

[42] S. Stieberger, (0,2) heterotic gauge couplings and their M-theory origin, Nucl. Phys. B 541 (1999) 109 [hep-th/9807124] [INSPIRE].

[43] R. Hlozek, D. Grin, D.J.E. Marsh and P.G. Ferreira, A search for ultralight axions using precision cosmological data, Phys. Rev. D 91 (2015) 103512 [arXiv:1410.2896] [InSPIRE].

[44] J.A. Harvey and G.W. Moore, Superpotentials and membrane instantons, hep-th/9907026 [INSPIRE].

[45] A. Arvanitaki, S. Dimopoulos, S. Dubovsky, N. Kaloper and J. March-Russell, String Axiverse, Phys. Rev. D 81 (2010) 123530 [arXiv:0905.4720] [InSPIRE]. 